\title{
Response to "Pharmacoinvasive strategy: An essential tool to avoid the Reperfusion Paradox in STEMI networks"
}

\author{
Respuesta a la carta al editor: "Estrategia farmacoinvasiva: herramienta para evitar la \\ Paradoja de Reperfusión en redes de infarto"
}

\author{
Rodrigo Gopar-Nieto*, Diego Araiza-Garaygordobil, and Alexandra Arias-Mendoza \\ Unidad Coronaria, Instituto Nacional de Cardiología Ignacio Chávez, Mexico City, Mexico
}

To the authors,

We are glad to read the comments about our article entitled "Demographic description and outcomes of a metropolitan network for myocardial infarction treatment"1. Our study describes and highlights the importance of integrating a metropolitan network to treat ST-segment elevation myocardial infarction, which remains a critical issue in low-to-middle income countries due to social, cultural, geographical, and logistical disparities. The full description of the 30-day outcomes in patients receiving either primary $\mathrm{PCl}$ or pharmacoinvasive strategy within our network is published elsewhere ${ }^{1}$. Regarding the questions raised by the authors, successful reperfusion after fibrinolytic was achieved in $59.1 \%$; however, the number of patients with contraindications to fibrinolysis was not recorded. Nevertheless, our complication rate was low ${ }^{2}$. We agree that door-to-needle time was higher than other similar studies, although we did not find significant differences in clinical outcomes. A possible explanation for this phenomenon is that education regarding STEMI standards of care, and access to diagnostic and therapeutic resources is still limited within the network. We must clarify that one of our main purposes is to educate health personnel to identify patients undergoing myocardial infarction, and since 2017, the national reperfusion program (PREMIA) has supported different educational and research initiatives to achieve such goal. Finally, we consider of utmost importance to communicate that the development of a STEMI network is feasible, even within a highly populated city in a middle-income country, and that outcomes within such networks are comparable with those of industrialized countries and well established reperfusion systems.

\section{References}

1. Gopar-Nieto R, Araiza-Garaygordobil D, Raymundo-Martínez GI, Martínez-Amezcua P, Cabello-López A, Manzur-Sandoval D, et al. Demographic description and outcomes of a metropolitan network for myocardial infarction treatment. Arch Cardiol Mex. 2021;91:167-77.

2. Araiza-Garaygordobil D, Gopar-Nieto R, Cabello-López A, Martinez-Amezcua P, Eid-Lidt G, Baeza-Herrera LA, Get al. Pharmacoinvasive strategy vs primary percutaneous coronary intervention in patients with st-elevation myocardial infarction: Results from a study in Mexico city. CJC Open 2020;3:409-18.
Available online: $29-10-2021$ Arch Cardiol Mex (Eng). 2021;91(4):544 www.archivoscardiologia.com license (http://creativecommons.org/licenses/by-nc-nd/4.0/) 